\title{
Beamforming for Rotated 3D Multipanel Array Structures for 5G NR MIMO Transmission
}

\author{
Hyukjun Lee, ${ }^{1}$ Wonjae Ryu, ${ }^{1}$ Wonjin Sung $\mathbb{D}^{1},{ }^{1}$ and Jonghyun Park ${ }^{2}$ \\ ${ }^{1}$ Department of Electronic Engineering, Sogang University, Seoul 04107, Republic of Korea \\ ${ }^{2}$ Advanced Standard R\&D Lab, LG Electronics, Seoul 07336, Republic of Korea \\ Correspondence should be addressed to Wonjin Sung; wsung@sogang.ac.kr
}

Received 26 December 2018; Revised 6 May 2019; Accepted 12 June 2019; Published 7 July 2019

Academic Editor: Symeon Nikolaou

Copyright (C) 2019 Hyukjun Lee et al. This is an open access article distributed under the Creative Commons Attribution License, which permits unrestricted use, distribution, and reproduction in any medium, provided the original work is properly cited.

5G new radio (NR) provides enhanced transmission capabilities to transceivers by utilizing the massive multiple-input multipleoutput (MIMO) technology with a significantly increased number of antenna elements. Such transmission requires massive arrays to perform accurate high-gain beamforming over the millimeter-wave frequency band. There is no fixed form of array structures for 5G NR base stations, but they are likely to include multiple subarrays or panels for practicality of implementation and are expected to cover the user equipment (UE) in various locations. In this paper, we propose an array structure to transmit signals over the three-dimensional (3D) space in an isotropic fashion for all types of UEs in ground, aerial, and high-rise building locations, by employing panels on surfaces of a polyhedron. We further derive exact beamforming equations for the proposed array and show the resulting beams provide improved receiver performance over the exiting conventional beamforming. The presented beamforming expressions can be applied to an arbitrary multipanel array with massive antenna elements.

\section{Introduction}

A massive multiple-input multiple-output (MIMO) technology enables signal transmission to multiple users at increased bandwidth efficiency, resulting in higher system capacity [1-4]. In 5G new radio (NR), the frequency band extends to the millimeter-wave to accommodate increased traffic over a larger bandwidth. Characteristics of the millimeterwave frequency band are important in designing transceivers and beamforming strategies, and related study has been conducted to attract a great amount of attention [4-6]. Larger propagation losses occurring at the millimeter-wave frequency carrier need to be compensated by beamforming techniques using a massive antenna array in order to have a sufficient spatial coverage. Shorter wavelengths of millimeterwave carriers reduce the size of each antenna element, making the utilization of arrays with a larger number of antenna elements practically feasible. Using such arrays, improved beamforming and beam management schemes can be performed [7].
A massive array can be decomposed into multiple subarrays in a smaller size for easier implementation, and studies have been conducted to propose efficient beamforming schemes for such subarray-based antenna structures [8-10]. Although full digital beamforming via baseband precoding provides accurate and controllable management of signal beams, it requires a separate RF chain to be connected to each antenna element which makes the array larger in size and more expensive to build. To overcome this problem, hybrid beamforming using analog beamforming to steer beams to the target direction for each subarray in addition to the digital precoding has been proposed [11, 12]. Subarrays are usually in the form of a uniform linear array (ULA) or a uniform rectangular array (URA), and beamforming vectors are required to steer beams to the desired direction. These beamforming vectors can be used as a part of baseband precoding for the case of full digital beamforming and applied to control phase shifters for the case of hybrid beamforming. Codebooks for ULAs and URAs have been studied for the limited feedback environment [13-15], and discrete Fourier 


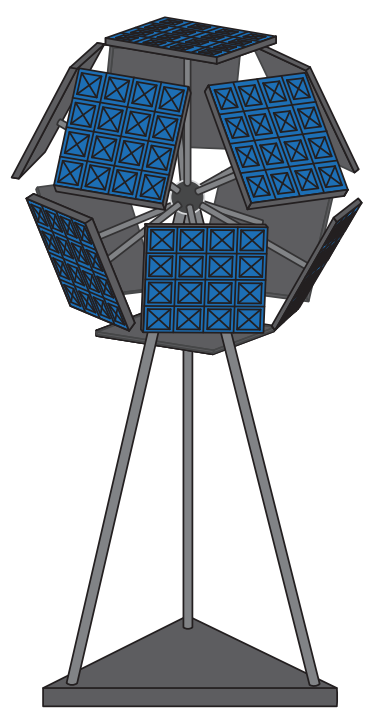

(a)

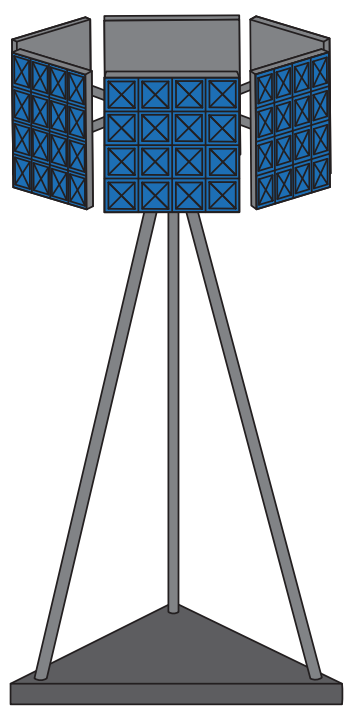

(b)

FIGURE 1: Antenna array structures: (a) polyhedron-based array and (b) hexagonal 6-sector array.

transform (DFT) based codebooks have been standardized to be used in 3GPP specifications [16, 17].

Attempts have been made to improve the beamforming and transmission efficiency by using antenna arrays in different shapes. In [18-20], uniform circular arrays (UCAs) to mitigate the interference at the sector boundary have been investigated, which are extended to the cylindrical shape to conduct vertical beamforming in addition to horizontal beamforming $[21,22]$. Vertical beamforming for small cells using the millimeter-wave has been studied in [23-25]. Signal transmission using spherically shaped arrays including geodesic domes was researched in [26-29]. Although an ultimate form of $3 \mathrm{D}$ arrays would be in spherical shape, such arrays do not only incur implementation complexity but cause difficulty to include subarrays. They also make the utilization of existing beamformers and codebooks difficult.

In this paper, we propose a $3 \mathrm{D}$ antenna array structure based on polyhedrons, which is composed of rectangular subarrays so that existing codebooks are applicable as a part of array beamforming. Due to the polyhedron-based nature of the array, it is capable of forming beams to all directions of the entire 3D space to cover a variety of moving objects as well as UEs with severe vertical tilting, both in upward and downward directions. A particular example of the polyhedron-based array in consideration is shown in Figure 1(a), where 12 rectangular subarrays are located on the surfaces of dodecahedron. The proposed antenna structure consisting of 12 URAs can be compared to the conventional hexagonal antenna structure with 6 subarrays shown in Figure 1(b), which is usually used to cover 6-sector cells. We use the method of coordinate transformation to derive exact beamforming equations for the proposed antenna structure and apply the resulting beamforming vectors to evaluate the transmission performance, to demonstrate the advantage of the proposal over the conventional array. Although the derivation and evaluation are conducted for the array as shown in the figure, the proposal can be generalized to many different forms of 3D arrays based on polyhedrons and geodesic structures.

The proposed BS architecture equipped with a massive array can increase the deployment cost for operators due to the hardware complexity required for the antennas and RF circuitry. This hardware cost, however, may be a small price to pay to obtain a high bandwidth efficiency and spatial multiplexing gain required for $5 \mathrm{G}$ radio access network (RAN) [30]. Since acquiring new spectrum and having them available for $5 \mathrm{G}$ NR is a key factor which increases the deployment cost, transceivers with the full utilization capability of the spectrum are desired [31, 32]. Furthermore, the subarray structure proposed here limits the number of RF chains to the number of subarrays, which maintains the cost of the radio unit at a reasonable level.

The paper is organized as follows. In Section 2, the system model is described and related parameters are introduced. In Section 3, the method of coordinate transmission is explained to combine subarray beamforming formulas for the $3 \mathrm{D}$ antenna structure, followed by the resulting derivation of beamforming equations for the proposed system in Section 4 . Beamforming performance in terms of the receiver power distribution is evaluated in Section 5, to quantify the amount of gain over the conventional case. Conclusions are given in Section 6.

\section{System Model}

We consider an antenna structure consisting of $M$ subarrays oriented in arbitrary directions in the $3 \mathrm{D}$ space. Each subarray includes $N_{1} \times N_{2}$ antenna elements with $N_{1}$ horizontal rows and $N_{2}$ vertical columns, thus the total number of antenna elements in the array becomes $M_{T}=M N_{1} N_{2}$. We assume that all antenna elements in the entire array are used to transmit a beam to the target user equipment (UE), 


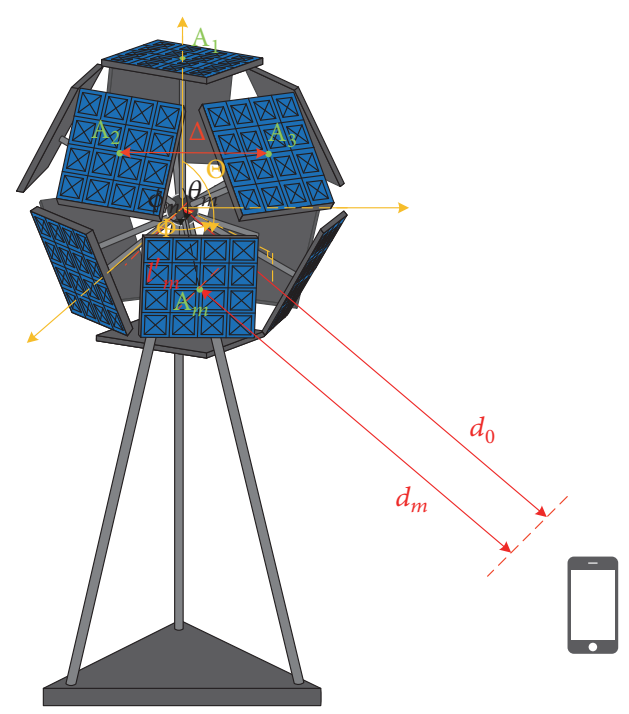

(a)

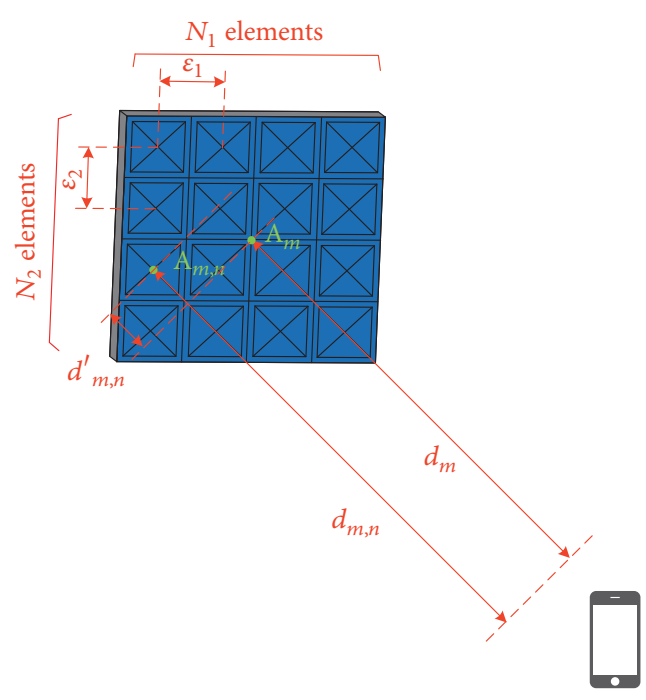

(b)

FIGURE 2: Antenna structure and parameters: (a) 12 subarrays and (b) single subarray.

requiring coherent combining of the signals from individual subarrays. Under the situation where only a subset of the subarrays is used form a beam to the target direction and the remaining subarrays are used for beams to other directions, the signal model used here can be modified accordingly. An extreme case of noncoherent operation is the $M$-sector model, for which each subarray is separately used to transmit the signal to the UE in its own sector.

Figure 2 shows the array structure with $4 \times 4$ rectangular subarrays located at the equal distance from the origin, i.e., the center of the whole array. The center point of the $m$-th surface of the dodecahedron is denoted by $\mathrm{A}_{m}, m=1,2, \ldots$, 12. As shown in Figure 2(a), the azimuth and zenith angles in polar coordinate which determine the location of $\mathrm{A}_{m}$ are denoted by $\phi_{m}$ and $\theta_{m}$, respectively. Also, the azimuth and zenith angles for the location of the target UE are denoted by $\Phi$ and $\Theta$, respectively. The distance between the UE and the origin is $d_{0}$, and the distance between $\mathrm{A}_{m}$ and the UE is $d_{m}$. In Figure 2(b), the location of the $n$-th element of the $m$-th subarray is denoted by $\mathrm{A}_{m, n}$, and the distance between adjacent elements is denoted by $\varepsilon_{1}$ for horizontal spacing and $\varepsilon_{2}$ for vertical spacing. The distance between $\mathrm{A}_{m, n}$ and the $\mathrm{UE}$ is $d_{m, n}$. The angles defined above are based on the global coordinate system (GCS), with the $x-y$ plane parallel to the ground and the $z$-axis vertically upward from the ground. To derive beamforming equations, we introduce the local coordinate system (LCS) for each subarray. The LCS for the $m$-th subarray is centered at $A_{m}$, with the subarray panel included in its $x-y$ plane and the $z$-axis directing the boresight of antenna elements. The azimuth and zenith angles of the UE location based on the LCS for the $m$-th array are denoted by $\Phi_{m}$ and $\Theta_{m}$, respectively. Parameter $R$ denotes the distance between the origin and $\mathrm{A}_{m}$ for all $\mathrm{m}$. Table 1 includes the parameters defined here and lists several more parameters used for the derivation of beamforming equations.
TABLE 1: Location and beam path parameters.

\begin{tabular}{lc}
\hline Symbols & Meaning \\
\hline $\mathrm{A}_{m}$ & Center point of the $m$-th subarray \\
$\mathrm{A}_{m, n}$ & $n$-th antenna element of $m$-th subarray \\
$\phi_{m}$ & Azimuth angle of $\mathrm{A}_{m}$ in GCS \\
$\theta_{m}$ & Zenith angle of $\mathrm{A}_{m}$ in GCS \\
$\Phi$ & UE azimuth angle in GCS \\
$\Theta$ & UE zenith angle in GCS \\
$\Phi_{m}$ & UE azimuth angle in LCS for the $m$-th subarray \\
$\Theta_{m}$ & UE zenith angle in LCS for the $m$-th subarray \\
$d_{0}$ & Distance between the origin and the UE \\
$d_{m}$ & Distance between $\mathrm{A}_{m}$ and the UE \\
$d_{m, n}$ & Distance between $\mathrm{A}_{m, n}$ and the UE \\
$d_{m, n}$ & $d_{m, n}-d_{m}$ \\
$l_{m}$ & $d_{m}-d_{0}$ \\
$l_{m, n}$ & $d_{m, n}-d_{0}\left(=l_{m}+d_{m, n}\right.$ ) \\
$R$ & Distance between the origin and the array center
\end{tabular}

The signal transmitted from the transmit antenna array of size $M_{T}$ to the receive antenna array of size $M_{R}$ can be represented by

$$
\mathbf{y}_{i}=\mathbf{H}_{i} \mathbf{W}_{i} \mathbf{s}_{i}+\sum_{j \neq i} \mathbf{H}_{i} \mathbf{W}_{j} \mathbf{s}_{j}+\mathbf{I}_{i}+\mathbf{n}_{i}
$$

where $\mathbf{y}_{i}=\left[\begin{array}{llll}y_{1} & y_{2} & \ldots & y_{M_{R}}\end{array}\right]^{T}$ is the received vector for the $i$-th UE and $\mathbf{H}_{i}=\left[\begin{array}{llll}\mathbf{h}_{1}{ }^{T} & \mathbf{h}_{2}{ }^{T} & \ldots & \mathbf{h}_{M_{R}}{ }^{T}\end{array}\right]^{T}$ is the $M_{R} \times M_{T}$ channel matrix with $\mathbf{h}_{r}=\left[\begin{array}{llll}h_{r, 1} & h_{r, 2} & \cdots & h_{r, M_{T}}\end{array}\right]$ denoting the channel vector for its $r$-th receive antenna. Precoding matrix $\mathbf{W}_{i}=\left[\begin{array}{llll}\mathbf{w}_{1} & \mathbf{w}_{2} & \cdots & \mathbf{w}_{K}\end{array}\right]$ includes beamforming vector $\mathbf{w}_{k}=$ $\left[\begin{array}{llll}w_{k, 1} & w_{k, 2} & \cdots & w_{k, M_{T}}\end{array}\right]^{T}$ for the $k$-th layer and $\mathbf{s}_{i}=$ 


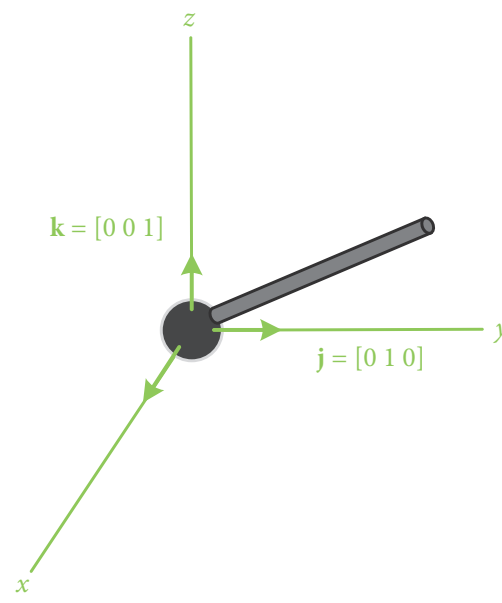

(a)

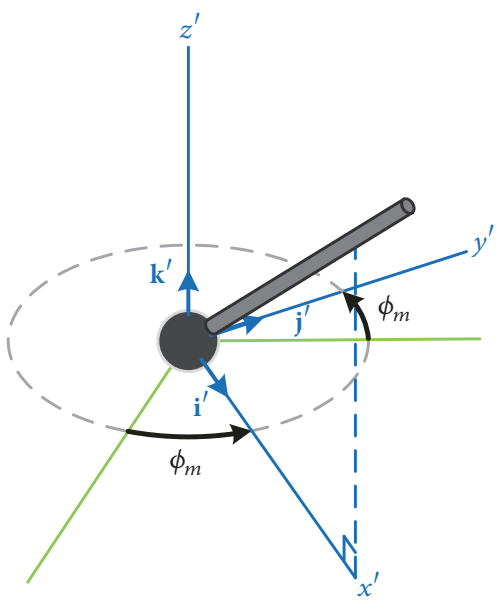

(b)

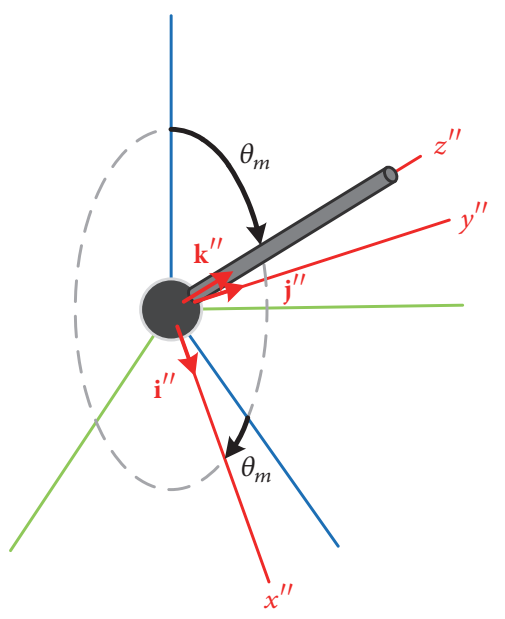

(c)

FIGURE 3: Coordinate transformation process.

$\left[\begin{array}{llll}s_{1} & s_{2} & \cdots & s_{K}\end{array}\right]^{T}$ is the transmitted data vector. Symbols $\mathbf{I}_{i}$ and $\mathbf{n}_{i}$ respectively denote $M_{R} \times 1$ vectors for the interference and additive white Gaussian noise (AWGN). For the singlerank transmission, precoding matrix $\mathbf{W}_{i}$ reduces to beamforming vector $\mathbf{w}=\left[\begin{array}{llll}w_{1} & w_{2} & \cdots & w_{M_{T}}\end{array}\right]^{T}$. Note that UE index $i$ is omitted in the beamforming vector symbol for notational simplicity.

The elements of $\mathbf{w}$ can be divided into $M$ groups of $N_{1} N_{2}$ elements as in $\mathbf{w}=\left[\begin{array}{llll}\mathbf{u}_{1}{ }^{T} & \mathbf{u}_{2}{ }^{T} & \cdots & \mathbf{u}_{M}{ }^{T}\end{array}\right]^{T}$, where $\mathbf{u}_{m}$ is the beamforming subvector for the $m$-th subarray determined as

$$
\mathbf{u}_{m}=u_{m} \mathbf{u}_{m}^{\prime}
$$

for $m=1,2, \ldots, M$. Here $u_{m}$ is a scalar parameter which compensates for the location of the $m$-th subarray within the whole array structure and determined by the relative distances of the subarray centers from the target UE. We obtain $u_{m}=\exp \left(-j 2 \pi l_{m} / \lambda\right)$, where $l_{m}$ is the beam path difference between $\mathrm{A}_{m}$ and the origin as shown in Figure 2(a) and $\lambda$ is the carrier wavelength. Then we construct vector $\mathbf{u}$ of compensation terms for all subarrays as

$$
\begin{aligned}
\mathbf{u} & =\left[\begin{array}{llll}
u_{1} & u_{2} & \cdots & u_{M}
\end{array}\right]^{T} \\
& =\frac{1}{\sqrt{M}}\left[\begin{array}{llll}
e^{-j(2 \pi / \lambda) l_{1}} & e^{-j(2 \pi / \lambda) l_{2}} & \cdots & e^{-j(2 \pi / \lambda) l_{M}}
\end{array}\right]^{T} .
\end{aligned}
$$

The vector component $\mathbf{u}_{m}^{\prime}$ in Equation (2) is the beamforming subvector for the $m$-th subarray without the location compensation and is expressed as

$$
\begin{aligned}
\mathbf{u}_{m}^{\prime} & =\left[\begin{array}{llll}
u_{1}^{(m)} & u_{2}^{(m)} & \cdots & u_{N_{1} N_{2}}^{(m)}
\end{array}\right]^{T} \\
& =\frac{1}{\sqrt{N_{1} N_{2}}}\left[\begin{array}{llll}
e^{-j(2 \pi / \lambda) d l_{m, 1}} & e^{-j(2 \pi / \lambda) d l_{m, 2}} & \cdots & e^{-j(2 \pi / \lambda) d l_{m, N_{1} N_{2}}}
\end{array}\right]^{T}
\end{aligned}
$$

where ${d^{\prime}}_{m, n}$ is the beam path difference $d_{m, n}-d_{m}$ as illustrated in Figure 2(b).

\section{Coordinate Transformation}

Coordinate transformation refers to the rotation of the axes of the GCS, to determine new axes to form the LCS for the $m$-th subarray. This process is needed to apply the existing beamformers and code vectors for the URA to the subarrays facing different directions in the $3 \mathrm{D}$ space. By performing the transformation, conventional beamforming vectors can easily be converted to find subvector $\mathbf{u}_{m}^{\prime}$ in Equation (4).

Figure 3 shows the coordinate transformation process via axis rotations in azimuth and zenith angles. In Figure 3(a), the unit vectors in the direction of $x, y$, and $z$ axes of the GCS, the original coordinate system, are respectively denoted by $\mathbf{i}$, $\mathbf{j}$, and $\mathbf{k}$. Rotation of the coordinate system by angle $\phi_{m}$ in the azimuth direction results in the movement of $x$ and $y$ axes in the in the $x-y$ plane, as shown in Figure 3(b), where the new axes are labeled as $x^{\prime}, y^{\prime}$, and $z^{\prime}$ axes. The unit vectors in the direction of the rotated axes are denoted as $\mathbf{i}^{\prime}, \mathbf{j}$ ', and $\mathbf{k}$. By the azimuth rotation, the unit vectors in two systems are related by the matrix linear transformation formula

$$
\left[\begin{array}{l}
\mathbf{i}^{\prime} \\
\mathbf{j}^{\prime} \\
\mathbf{k}^{\prime}
\end{array}\right]=\left[\begin{array}{ccc}
\cos \phi_{m} & \sin \phi_{m} & 0 \\
-\sin \phi_{m} & \cos \phi_{m} & 0 \\
0 & 0 & 1
\end{array}\right]\left[\begin{array}{l}
\mathbf{i} \\
\mathbf{j} \\
\mathbf{k}
\end{array}\right]
$$

where it can be verified that $\mathbf{k}^{\prime}=\mathbf{k}$ since the $z$-axis does not change due to this rotation. The second rotation of the coordinate system is in the zenith direction by angle $\theta_{m}$, resulting in the movement of $x^{\prime}$ and $z^{\prime}$ axes in the $x^{\prime}-z^{\prime}$ plane. The new coordinate axes are named $x^{\prime \prime}, y^{\prime \prime}$, and $z^{\prime \prime}$ as indicated in Figure 3(c). The corresponding unit vectors along these axes are denoted by i", $\mathbf{j}$ ", $\mathbf{k}$ " and can be determined by the second matrix transformation formula

$$
\left[\begin{array}{c}
\mathbf{i}^{\prime \prime} \\
\mathbf{j}^{\prime \prime} \\
\mathbf{k}^{\prime \prime}
\end{array}\right]=\left[\begin{array}{ccc}
\cos \theta_{m} & 0 & -\sin \theta_{m} \\
0 & 1 & 0 \\
\sin \theta_{m} & 0 & \cos \theta_{m}
\end{array}\right]\left[\begin{array}{l}
\mathbf{i}^{\prime} \\
\mathbf{j}^{\prime} \\
\mathbf{k}^{\prime}
\end{array}\right]
$$


for which $\mathbf{j}^{\prime \prime}=\mathbf{j}$ ' holds due to no movement of the $y^{\prime}$ axis. Combining (5) and (6), we obtain

$$
\begin{aligned}
& {\left[\begin{array}{l}
\mathbf{i}^{\prime \prime} \\
\mathbf{j}^{\prime \prime} \\
\mathbf{k}^{\prime \prime}
\end{array}\right]} \\
& \quad=\left[\begin{array}{ccc}
\cos \theta_{m} \cos \phi_{m} & \cos \theta_{m} \sin \phi_{m} & -\sin \theta_{m} \\
-\sin \phi_{m} & \cos \phi_{m} & 0 \\
\sin \theta_{m} \cos \phi_{m} & \sin \theta_{m} \sin \phi_{m} & \cos \theta_{m}
\end{array}\right]\left[\begin{array}{l}
\mathbf{i} \\
\mathbf{j} \\
\mathbf{k}
\end{array}\right],
\end{aligned}
$$

and unit vectors i", j", and k" can be written as

$$
\begin{aligned}
\mathbf{i}^{\prime \prime} & =\left[\cos \theta_{m} \cos \phi_{m}, \cos \theta_{m} \sin \phi_{m},-\sin \theta_{m}\right], \\
\mathbf{j}^{\prime \prime} & =\left[-\sin \phi_{m}, \cos \phi_{m}, 0\right], \\
\mathbf{k}^{\prime \prime} & =\left[\sin \theta_{m} \cos \phi_{m}, \sin \theta_{m} \sin \phi_{m}, \cos \theta_{m}\right] .
\end{aligned}
$$

The azimuth and zenith angles indicating the position of the UE are $\Phi$ and $\Theta$ as given in Table 1. Denoting the UE distance from the origin by $\rho$, the Cartesian coordinate of the UE in the GCS is represented by vector

$$
\mathbf{e}=[\rho \cos \Phi \sin \Theta, \rho \sin \Phi \sin \Theta, \rho \cos \Theta]
$$

Using the coordinate transformation, we can represent the UE location in the LCS for the $m$-th subarray by the new Cartesian coordinate $\mathbf{e}_{m}=[\alpha, \beta, \gamma]$, which is the vector from $\mathrm{A}_{m}$ to the UE. The elements of $\mathbf{e}_{m}$ can be obtained by

$$
\begin{aligned}
& \alpha=\mathbf{e} \cdot \mathbf{i}^{\prime \prime}, \\
& \beta=\mathbf{e} \cdot \mathbf{j}^{\prime \prime}, \\
& \gamma=\mathbf{e} \cdot \mathbf{k}^{\prime \prime}
\end{aligned}
$$

where $(\bullet)$ represents the dot product of two vectors. Using (8), (9), and (10), the element values are calculated as

$$
\begin{aligned}
\alpha & =\rho\left(\cos \theta_{\mathrm{m}} \cos \phi_{m} \cos \Phi \sin \Theta\right. \\
& \left.+\cos \theta_{\mathrm{m}} \sin \phi_{m} \sin \Phi \sin \Theta-\sin \theta_{\mathrm{m}} \cos \Theta\right) \\
& =\rho\left(\cos \theta_{\mathrm{m}} \sin \Theta \cos \left(\Phi-\phi_{m}\right)-\sin \theta_{\mathrm{m}} \cos \Theta,\right. \\
\beta & =\rho\left(-\sin \phi_{m} \cos \Phi \sin \Theta+\cos \phi_{m} \sin \Phi \sin \Theta\right)=\rho \\
& \cdot \sin \Theta \sin \left(\Phi-\phi_{m}\right), \\
\gamma & =\rho\left(\sin \theta_{\mathrm{m}} \cos \phi_{m} \cos \Phi \sin \Theta\right. \\
& \left.+\sin \theta_{\mathrm{m}} \sin \phi_{m} \sin \Phi \sin \Theta+\cos \theta_{\mathrm{m}} \cos \Theta\right) \\
& =\rho\left(\sin \theta_{\mathrm{m}} \sin \Theta \cos \left(\Phi-\phi_{m}\right)+\cos \theta_{\mathrm{m}} \cos \Theta\right) .
\end{aligned}
$$

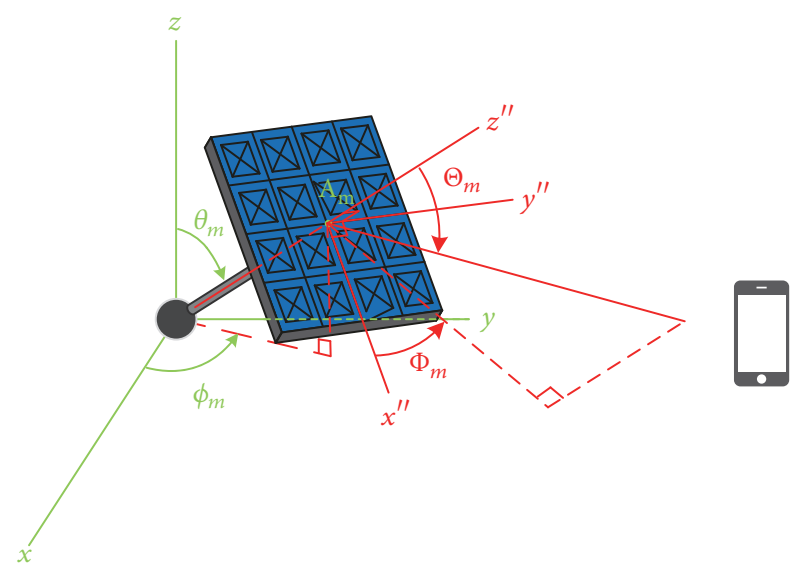

Figure 4: Angle parameters for the GCS and the LCS.

From the calculation results, zenith angle $\Theta_{m}$ and azimuth angle $\Phi_{m}$ of the UE in the LCS for the $m$-th subarray can be determined as

$$
\begin{aligned}
\Theta_{\mathrm{m}} & =\cos ^{-1} \frac{\gamma}{\rho} \\
& =\cos ^{-1}\left(\sin \theta_{\mathrm{m}}\right. \\
& \left.\cdot \sin \Theta \cos \left(\Phi-\phi_{m}\right)+\cos \theta_{\mathrm{m}} \cos \Theta\right), \\
\Phi_{\mathrm{m}} & =\tan ^{-1} \frac{\beta}{\alpha} \sin \Theta \sin \left(\Phi-\phi_{m}\right) \\
& =\tan ^{-1}\left(\frac{\sin \theta_{\mathrm{m}} \cos \Theta}{\cos \theta_{\mathrm{m}} \sin \Theta \cos \left(\Phi-\phi_{m}\right)-\sin }\right) .
\end{aligned}
$$

These transformed angles obtained by the coordinate transformation are illustrated in Figure 4. Also, the unit vector in the direction of $\mathbf{e}_{m}$ is defined as $\mathbf{f}_{m}=\mathbf{e}_{m} /\left|\mathbf{e}_{m}\right|$, expressed by

$$
\mathbf{f}_{m}=\left[\cos \Phi_{m} \sin \Theta_{m}, \sin \Phi_{m} \sin \Theta_{m}, \cos \Theta_{m}\right] \text {. }
$$

The unit vector in (13) indicating the direction of the UE in the LCS can be used to determine the beam path difference for different antenna elements in the $m$-th subarray. The antenna elements represented in the Cartesian coordinate exist on the $x "-y$ " plane of the LCS and are given by $\mathrm{A}_{m, 1}=\left(x_{m, 1}, y_{m, 1}, 0\right), \mathrm{A}_{m, 2}=\left(x_{m, 2}, y_{m, 2}, 0\right), \cdots, \mathrm{A}_{m, N_{1} N_{2}}=$ $\left(x_{m, N_{1} N_{2}}, y_{m, N_{1} N_{2}}, 0\right)$.

We further denote the vector originating from $\mathrm{A}_{m}$ and ending at $\mathrm{A}_{m, n}$ by

$$
\mathbf{a}_{m, n}=\left[x_{m, n}, y_{m, n}, 0\right]
$$

for $m=1,2, \ldots, N_{1} N_{2}$. Using these location vectors $\left\{\mathbf{a}_{m, n}\right\}$ for antenna elements and the direction vector $\mathbf{f}_{m}$ for the UE in (13), we derive the path difference and the compensation terms for the phase rotation.

\section{Subarray Beamforming}

To obtain beamforming subvector $\mathbf{u}_{m}$ for the $m$-th subarray, we use positioning vectors in the LCS to determine the beam 
path difference for each of the antenna arrays. As shown in Figure 5, vector $\mathbf{a}_{m, n}$ points antenna element $A_{m, n}$ from subarray center $\mathrm{A}_{m}$, and vector $\mathbf{f}_{m}$ points the target UE from subarray center $\mathrm{A}_{m}$. The difference $d_{m, n}-d_{m}$ of the beam distance from each antenna element to the UE, with the array center $\mathrm{A}_{m}$ as the reference point, can be calculated as

$$
d_{m, n}^{\prime}=-\mathbf{a}_{m, n} \cdot \mathbf{f}_{m} .
$$

Using (13), (14), and (15), we obtain

$$
\begin{aligned}
& d_{m, n}^{\prime} \\
& \quad=-\left(x_{m, n} \sin \Theta_{\mathrm{m}} \cos \Phi_{\mathrm{m}}+y_{m, n} \sin \Theta_{\mathrm{m}} \sin \Phi_{\mathrm{m}}\right) .
\end{aligned}
$$

The difference of the beam distance causes the phase rotation of the transmit/receive signals, which needs to be compensated by the beamforming subvector $\mathbf{u}_{m}^{\prime}$. The phase components of the subvector elements are expressed as

$$
\angle \mathbf{u}_{m}^{\prime}=-\frac{2 \pi}{\lambda}\left[d_{m, 1}^{\prime}, d_{m, 2}^{\prime}, \ldots, d_{m, N_{1} N_{2}}^{\prime}\right]^{T} .
$$

As discussed in Section 2, the beamforming subvector $\mathbf{u}_{m}$ including the subarray location compensation term $u_{m}$ is represented as $\mathbf{u}_{m}=u_{m} \mathbf{u}_{m}^{\prime}$, and its phase components can be written as

$$
\angle \mathbf{u}_{m}=-\frac{2 \pi}{\lambda}\left[l_{m, 1}, l_{m, 2}, \ldots, l_{m, N_{1} N_{2}}\right]^{T}
$$

where $l_{m, n}=d_{m, n}-d_{0}$ is the difference of the beam distances from $\mathrm{A}_{m, n}$ and from the origin. The path difference $l_{m, n}$ can be described as the sum

$$
l_{m, n}=l_{m}+d_{m, n}^{\prime}
$$

where $l_{m}=d_{m}-d_{0}$ is the difference of the beam distances from the subarray center $A_{m}$ and from the origin. Using Equation (3.1) of [33], we obtain

$$
l_{m}=-R\left(\sin \Theta \sin \theta_{m} \cos \left(\Phi-\Phi_{m}\right)+\cos \Theta \cos \theta_{m}\right) .
$$

Combining Equations (16), (19), and (20), the path difference $l_{m, n}$ is computed as

$$
\begin{aligned}
l_{m, n}= & -R\left(\sin \Theta \sin \theta_{m} \cos \left(\Phi-\Phi_{m}\right)+\cos \Theta \cos \theta_{m}\right) \\
& -\left(x_{m, n} \sin \Theta_{\mathrm{m}} \cos \Phi_{\mathrm{m}}+y_{m, n} \sin \Theta_{\mathrm{m}} \sin \Phi_{\mathrm{m}}\right)
\end{aligned}
$$

which can be applied to (18) to determine $\mathbf{u}_{m}$. Finally, the beamforming vector for the entire array including all subarrays is obtained as $\mathbf{w}=\left[\begin{array}{lllll}\mathbf{u}_{1}{ }^{T} & \mathbf{u}_{2}{ }^{T} & \cdots & \mathbf{u}_{M}{ }^{T}\end{array}\right]^{T}$ by using

$$
\mathbf{u}_{m}=\left[e^{-(2 \pi / \lambda) l_{m, 1}}, e^{-(2 \pi / \lambda) l_{m, 2}}, \ldots, e^{-(2 \pi / \lambda) l_{m, N_{1} N_{2}}}\right]^{T}
$$

As an example, consider subarrays of size $2 \times 2$ with interelement spacing $\varepsilon_{1}=\varepsilon_{2}=\varepsilon$ applied to the dodecahedron structure in Figure 2(a). In this case, position vectors for antenna elements in the $m$-th subarray are $\mathbf{a}_{m, 1}=$ $[-\varepsilon / 2, \varepsilon / 2,0], \mathbf{a}_{m, 2}=[\varepsilon / 2, \varepsilon / 2,0], \mathbf{a}_{m, 3}=[\varepsilon / 2,-\varepsilon / 2,0]$, and $\mathbf{a}_{m, 4}=[-\varepsilon / 2,-\varepsilon / 2,0]$. Using our result in (21), the phase components of the beamforming subvector $\mathbf{u}_{m}$ are given as

$$
\angle \mathbf{u}_{m}=-\frac{2 \pi}{\lambda}\left[\begin{array}{l}
-R\left(\sin \Theta \sin \theta_{m} \cos \left(\Phi-\Phi_{m}\right)+\cos \Theta \cos \theta_{m}\right)-\left(\frac{\varepsilon}{2}\right)\left(-\sin \Theta_{m} \cos \Phi_{m}+\sin \Theta_{m} \sin \Phi_{m}\right) \\
-R\left(\sin \Theta \sin \theta_{m} \cos \left(\Phi-\Phi_{m}\right)+\cos \Theta \cos \theta_{m}\right)-\left(\frac{\varepsilon}{2}\right)\left(+\sin \Theta_{m} \cos \Phi_{m}+\sin \Theta_{m} \sin \Phi_{m}\right) \\
-R\left(\sin \Theta \sin \theta_{m} \cos \left(\Phi-\Phi_{m}\right)+\cos \Theta \cos \theta_{m}\right)-\left(\frac{\varepsilon}{2}\right)\left(+\sin \Theta_{m} \cos \Phi_{m}-\sin \Theta_{m} \sin \Phi_{m}\right) \\
-R\left(\sin \Theta \sin \theta_{m} \cos \left(\Phi-\Phi_{m}\right)+\cos \Theta \cos \theta_{m}\right)-\left(\frac{\varepsilon}{2}\right)\left(-\sin \Theta_{m} \cos \Phi_{m}-\sin \Theta_{m} \sin \Phi_{m}\right)
\end{array}\right]
$$

which can be applied to (22). Beamforming vector $\mathbf{w}$ is then obtained to coherently direct the signal to the target UE.

\section{Evaluation}

To evaluate the beamforming performance, we use two different UE distribution models shown in Figure 6. The first model is the conventional two-dimensional (2D) UE distribution within a cell shown in Figure 2(a). We consider the cell radii in the range from 20 to $100 \mathrm{~m}$ to reflect the small cell nature of the $5 \mathrm{G}$ NR transmission. The second model shown in Figure 2(b) uses the 3D UE distribution which may become more prominent in the near future, with the advent of various moving internet-of-things (IoT) devices such as drones. For both models, UEs are randomly generated in a uniform fashion within the defined range of appearance, i.e., inside the circle for the $2 \mathrm{D}$ model and on the surface of the sphere for the $3 \mathrm{D}$ model. Performance is evaluated for the proposed dodecahedron structure with 12 subarrays of size $4 \times 4$ shown in Figure 1(a), which is compared to that of the hexagonal structure with 6 subarrays of the same $4 \times 4$ size shown in Figure 1(b). For both array structures, the height 


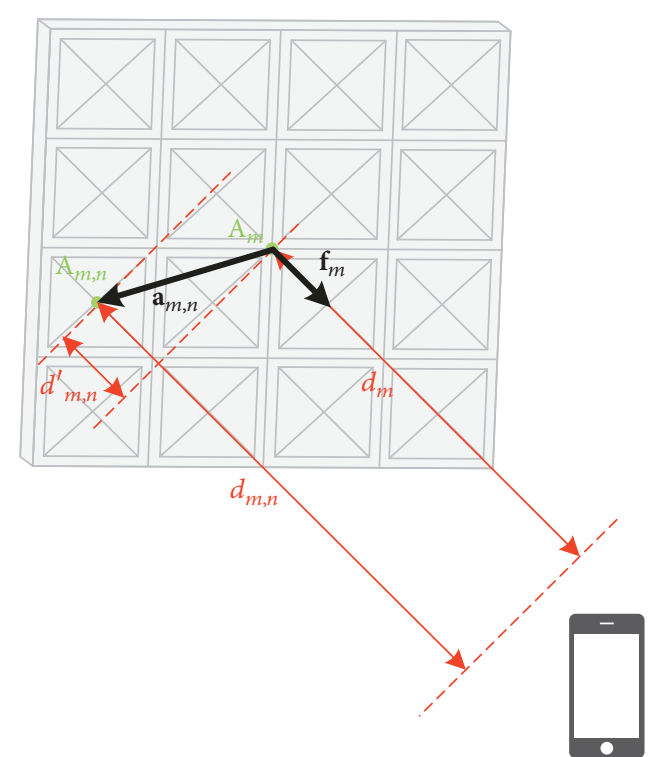

FIGURE 5: Inner product of two positioning vectors.

of the array, from the ground level to the center of the array, is set to $25 \mathrm{~m}$. The height of the UE in $2 \mathrm{D}$ model is set to $1.5 \mathrm{~m}$.

Although both the large- and small-scale fading are important factors determining the link performance of wireless communication systems, the focus of evaluation in this section is the effect of the line-of-sight (LoS) component for the array structures in comparison. This can be justified by the fact that we are more interested in understanding the performance for small cell environments utilizing millimeterwave with the sparsity of few dominant paths. The effect of the LoS component is especially important in the $3 \mathrm{D}$ model, for which less reflectors exist for the upper hemisphere region.

The deviation angle and the normalized received power are used as key performance measures. The deviation angle is the angle between the boresight of each subarray panel and the target UE. The received power is expected to increase for a smaller value of the deviation angle, due to the antenna gain pattern of the subarray. For a given location of the target UE, deviation angles can be determined for all $M$ subarrays which are then sorted in an ascending order, denoted by symbols $\Theta^{(1)}, \Theta^{(2)}, \ldots, \Theta^{(M)}$. The normalized received power from each subarray is determined by the corresponding deviation angle and the antenna gain pattern, with $0 \mathrm{~dB}$ denoting the maximum power for the UE located at the exact boresight of the subarray panel. The normalized received power values from all subarrays are sorted in a descending order as $P_{1}, P_{2}, \ldots, P_{M}$. The antenna gain pattern follows the $3 \mathrm{GPP}$ model in [34] given as

$$
G\left(\varphi_{v}, \varphi_{h}\right)=-\min \left\{-\left[G_{v}\left(\varphi_{v}\right)+G_{h}\left(\varphi_{h}\right)\right], 30\right\}[\mathrm{dB}]
$$

where $G_{v}\left(\varphi_{v}\right)=-\min \left\{\left(\varphi_{v} / 65^{\circ}\right)^{2}, 30\right\}$ and $G_{h}=$ $-\min \left\{\left(\varphi_{h} / 65^{\circ}\right)^{2}, 30\right\}$ are respectively called the vertical and horizontal gains. Arguments $\varphi_{v}$ and $\varphi_{h}$ represent the vertical and horizontal deviation angles from the antenna boresight.
Figure 7 shows the performance comparison results for the proposed array structure and the conventional hexagonal array structure using the 2D UE distribution model with cell radii 20,40 , and $60 \mathrm{~m}$. The probability density function (PDF) of deviation angle $\Theta^{(1)}$ from the most significant subarray panel is indicated in Figure 7(a) for two structures. It can be verified from the figure that the deviation angle for the proposed array becomes substantially smaller than that of the conventional array as the cell radius decreases. This is due to the structural advantage of the proposal for downward beamforming to UEs located close to the base station. When the cell radius is $20 \mathrm{~m}$, the average deviation angle difference between two arrays is $36^{\circ}$, which results in a great amount of difference in the received power as shown in Figure 7(b). The PDF of the normalized received power $P_{1}$ from the most significant subarray is obtained from $\Theta^{(1)}$ and the antenna gain formula in (24). As can be seen from the figure, the power distribution for the hexagonal array is clearly shifted to the lower Rx levels, providing inferior receiver performance compared to the case of proposed array. The performance difference between two arrays is more striking for receiver locations close to the base; for the cell radius of $20 \mathrm{~m}$, the received power distribution for the hexagonal array ranges from -25 to $-7 \mathrm{~dB}$, in comparison to -20 to $0 \mathrm{~dB}$ for the polyhedron-based array. The average power difference amounts to $10 \mathrm{~dB}$ in this case. Performance advantage of the proposal can be seen for other cell radius vales, and the average gain of about $7 \mathrm{~dB}$ is obtained for three radius values used for performance evaluation.

In Figure 8, the average of normalized received power values is shown for the $2 \mathrm{D}$ cell radius of 20 to $100 \mathrm{~m}$. Although the performance gap between two array structures decreases as the cell size increases, a significant amount of power gain can be observed over the entire range of cell radii. Digital beamforming only in the figure refers to the case when subarray beam tilting is not applied, showing a severe performance degradation compared to the hybrid beamforming with full phase compensation described in (22) applied. The amount of power gain of the proposed structure over the conventional array is in the range from 5 to $10 \mathrm{~dB}$ when hybrid beamforming is used for cell size $60 \mathrm{~m}$ or less. For digital beamforming only case, the gain ranges from 8 to $15 \mathrm{~dB}$.

The performance comparison results based on the $3 \mathrm{D}$ UE distribution model are shown in Figure 9. The PDF of deviation angle $\Theta^{(1)}$ for the proposed array structure is more highly concentrated in smaller values, resulting in higher received power values. The conventional array has a widespread power distribution for the 3D UE model, producing very large deviation angle values and thus small received power for a significant portion of UEs. On the average, the deviation angle decreased by $16^{\circ}$ and the received power increases by $4 \mathrm{~dB}$ by using the dodecahedron array.

Assumptions and approximations considered here can be either logically justified or experimentally verified as follows. First, the channel model with the dominant LoS path has been put into use for MIMO transmission under various 


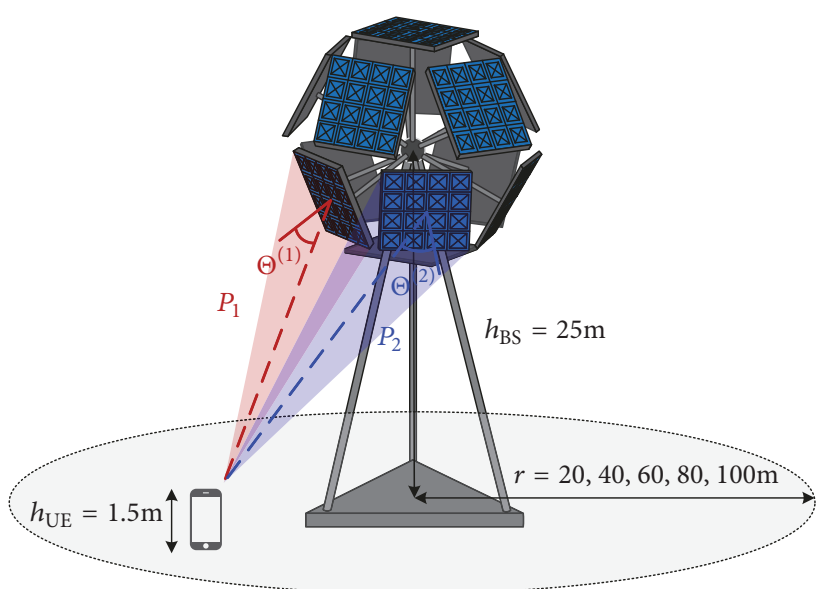

(a)

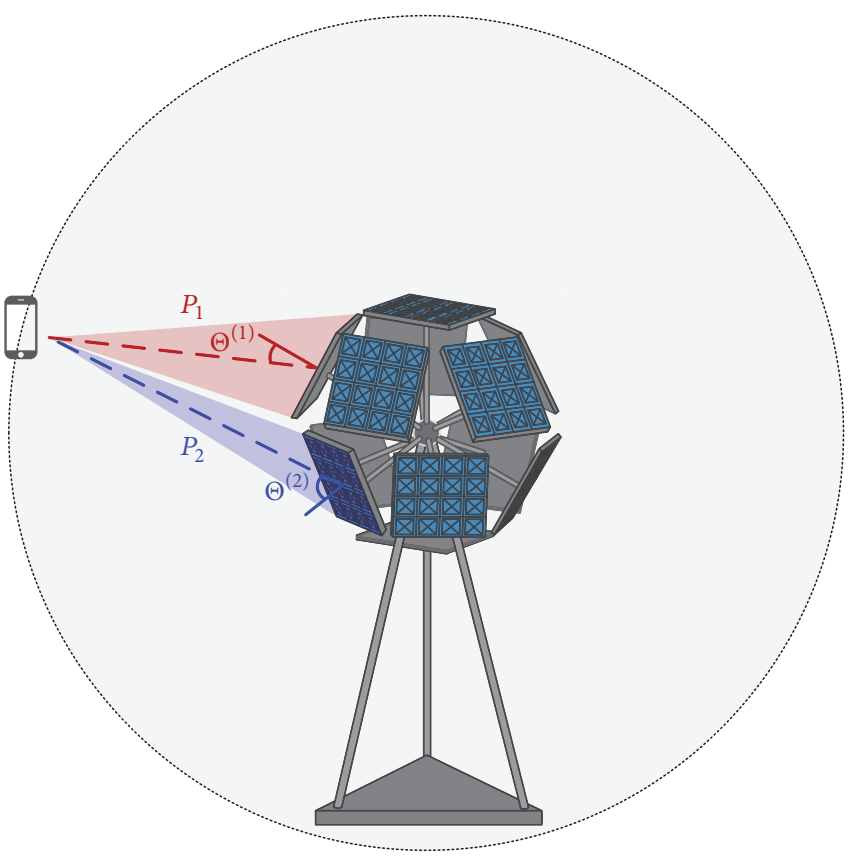

(b)

FIGURE 6: : Performance evaluation models: (a) 2D UE distribution and (b) 3D UE distribution.

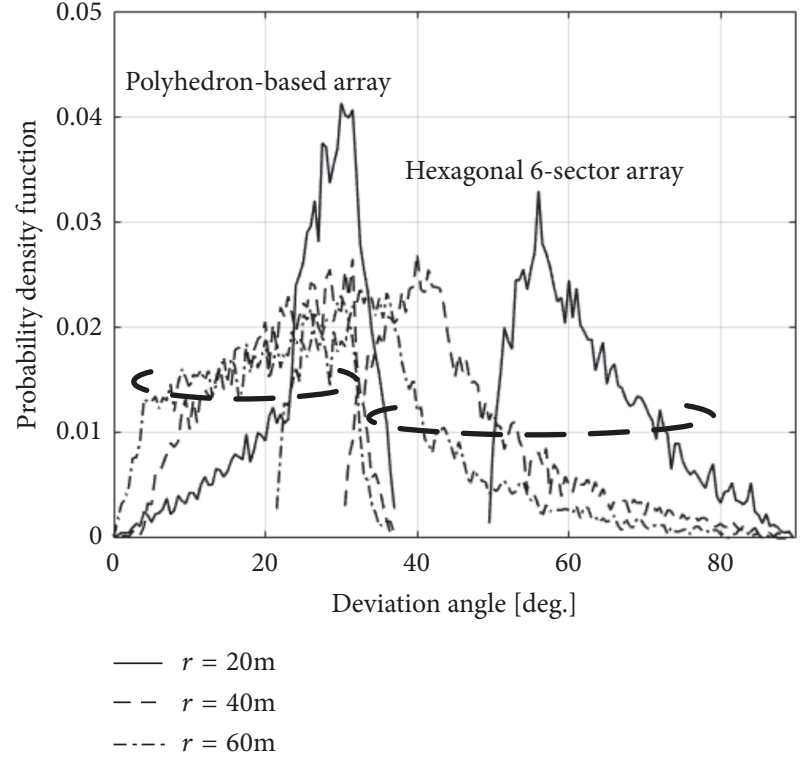

(a)

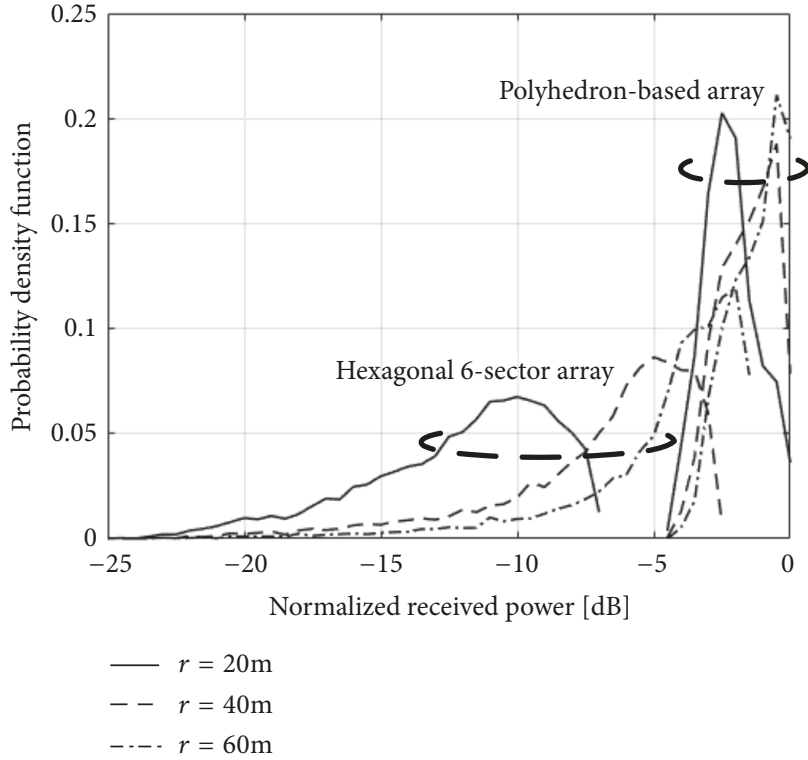

(b)

FIGURE 7: Performance for the 2D UE distribution: (a) deviation angles and (b) the received power.

circumstances including the 3D channel $[35,36]$ and its empirical verification has been performed to validate the usefulness of the model using the measured field data over a wide range of the carrier frequency [37]. Comparison of the measured and modelled data has also been made using a prototype to confirm the accuracy of the model in [38]. Second, the antenna gain pattern in (24) which we applied for performance evaluation has been adopted by the 3GPP specification after the field test and calibration of multiple vendor sources [34]. The uniform UE distribution assumption is widely used for cellular performance evaluation as in the standard document for the 5G NR radio access network [39]. The hybrid beamforming operation via digital beam generation and analog beam tilting considered here is one of the most commonly proposed methods for massive MIMO transmission as discussed in $[11,12,40]$. 


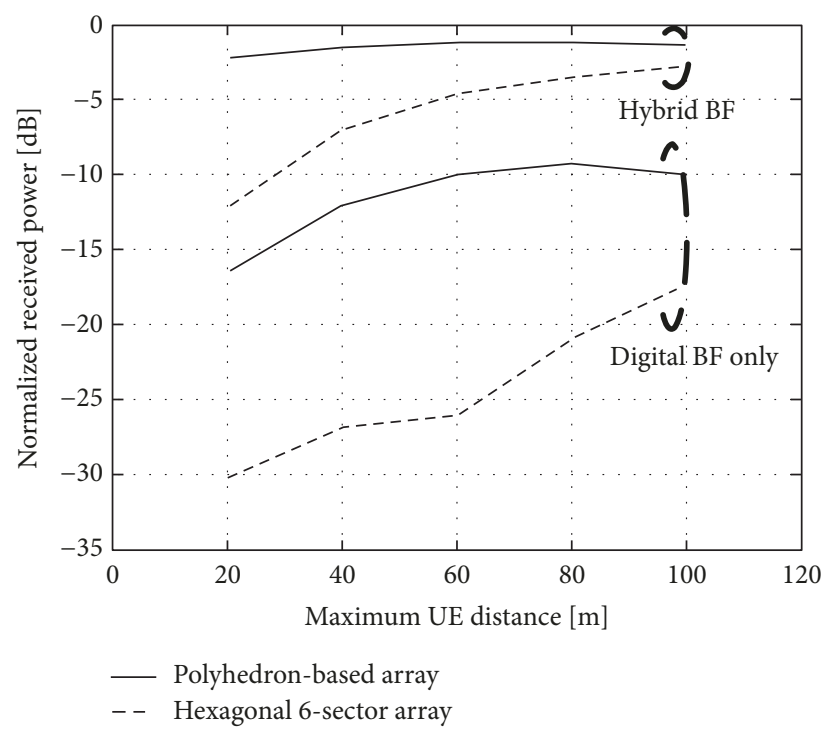

FIGURE 8: Average received power for the 2D UE distribution.

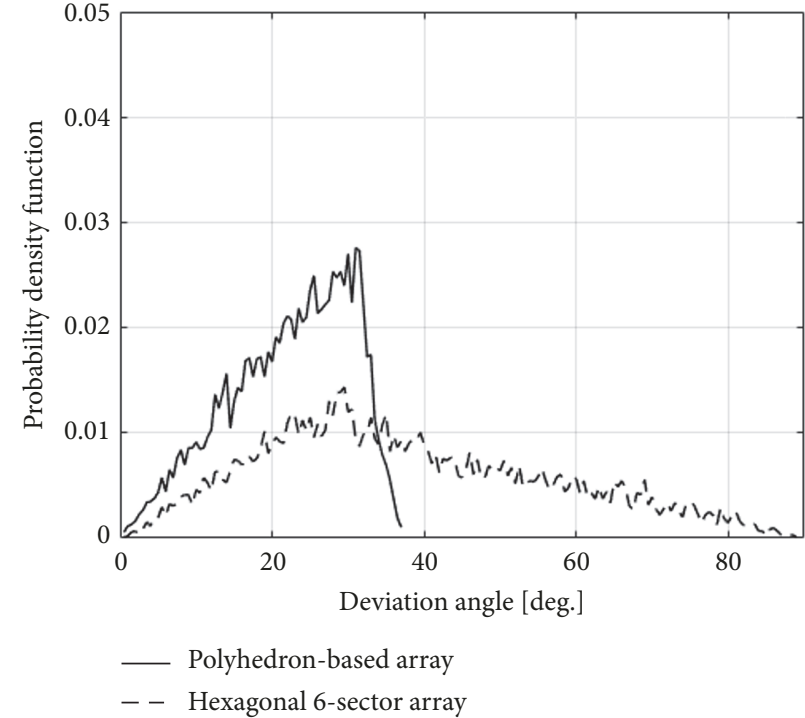

(a)

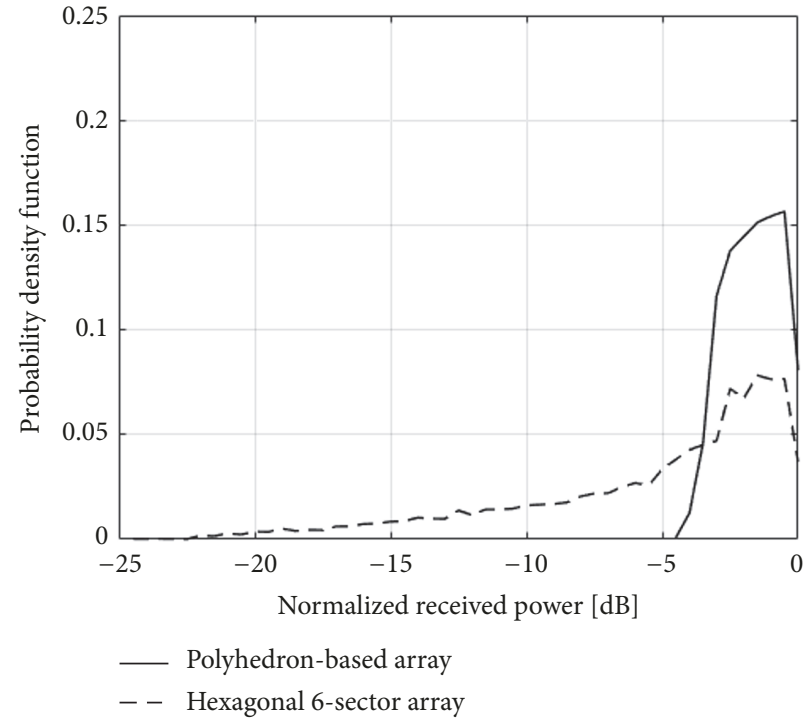

(b)

FIGURE 9: Performance for the 3D UE distribution: (a) deviation angles and (b) the received power.

\section{Conclusions}

A new class of antenna array structures with subarray panels on polyhedrons is proposed to cope with wider UE distributions in the $3 \mathrm{D}$ space, and a design example based on the dodecahedron is discussed. Presented beamforming equations can be applied to various antenna structures with subarrays facing arbitrary directions. For both the small cell and 3D UE distribution models, our proposal provides desirable performance measures as confirmed by the simulation results.

\section{Data Availability}

Underlying data related to this article has been originally obtained by the authors using the simulation package of
Digital Transmission Lab, Sogang University, and is available upon request.

\section{Conflicts of Interest}

The authors declare that they have no conflicts of interest.

\section{Acknowledgments}

This work was supported by the National Research Foundation (NRF) of Korea, MSIP (NRF-2017R1A2B4002367) and by LG Electronics. 


\section{References}

[1] Y. Dai, S. Jin, L. Jiang, X. Gao, and M. Lei, "A PMI feedback scheme for downlink multi-user MIMO based on dualcodebook of LTE-Advanced," in Proceedings of the VTC-Fall '12, pp. 1-5, Quebec City, Canada, September 2012.

[2] K. Schober, M. Enescu, and R. Wichman, "MIMO adaptive codebook for cross-polarized antenna arrays," in Proceedings of the 2013 IEEE 24th Annual International Symposium on Personal, Indoor, and Mobile Radio Communications, PIMRC 2013, pp. 1125-1129, London, UK, September 2013.

[3] L. Lu, G. Y. Li, A. L. Swindlehurst, A. Ashikhman, and R. Zhang, "An overview of massive MIMO: benefits and challenges," IEEE Journal of Selected Topics in Signal Processing, vol. 8, no. 8, pp. 742-758, 2014.

[4] S. Sun, T. S. Rappaport, R. W. Heath, A. Nix, and S. Rangan, "MIMO for millimeter-wave wireless communications: beamforming, spatial multiplexing, or both?" IEEE Communications Magazine, vol. 52, no. 12, pp. 110-121, 2014.

[5] A. Swindlehurst, E. Ayanoglu, P. Heydari, and F. Capolino, "Millimeter-wave massive MIMO: the next wireless revolution?" IEEE Communications Magazine, vol. 52, no. 9, pp. 56-62, 2014.

[6] L. Wei, R. Hu, Y. Qian, and G. Wu, "Key elements to enable millimeter wave communications for 5G wireless systems," IEEE Wireless Communications Magazine, vol. 21, no. 6, pp. 136143, 2014.

[7] E. Onggoanusi, S. Rahman, L. Guo et al., "Modular and highresolution channel state information and beam management for $5 \mathrm{G}$ new radio," IEEE Communications Magazine, vol. 56, no. 3, pp. 48-55, 2018.

[8] L. Zhou and Y. Ohashi, "Fast codebook-based beamforming training for mmWave MIMO systems with subarray structures," in Proceedings of the 2015 IEEE 82nd Vehicular Technology Conference (VTC Fall), pp. 1-5, Boston, Mass, USA, September 2015.

[9] J. Zhang, Y. Huang, C. Zhang, S. He, M. Xiao, and L. Yang, "Cooperative multi-subarray beam training in millimeter wave communication systems," in Proceedings of the 2017 IEEE Global Communications Conference (GLOBECOM 2017), pp. 16, Singapore, December 2017.

[10] Y. S. Faouri, R. Hussain, and M. S. Sharawi, "A 4-element subarray with 4-polarization modes," in Proceedings of the 2017 IEEE Jordan Conference on Applied Electrical Engineering and Computing Technologies (AEECT), pp. 1-4, Aqaba, October 2017.

[11] H. Seleem, A. I. Sulyman, and A. Alsanie, "Hybrid precodingbeamforming design with hadamard RF codebook for mmWave large-scale MIMO systems," IEEE Access, vol. 5, pp. 6813-6823, 2017.

[12] A. Alkhateeb, J. Mo, N. González-Prelcic, and R. W. Heath Jr., "MIMO precoding and combining solutions for millimeterwave systems," IEEE Communications Magazine, vol. 52, no. 12, pp. 122-131, 2014.

[13] X. Su, J. Zeng, J. Li et al., "Limited feedback precoding for massive MIMO," International Journal of Antennas and Propagation, vol. 2013, pp. 1-9, 2013.

[14] Y. Han, S. Jin, X. Li, Y. Huang, L. Jiang, and G. Wang, "Design of double codebook based on 3D dual-polarized channel for multiuser MIMO system," EURASIP Journal on Advances in Signal Processing, vol. 2014, no. 1, 2014.

[15] F. W. Vook, E. Visotsky, T. A. Thomas, and B. Mondal, "Product codebook feedback for massive MIMO with cross-polarized 2D antenna arrays," in Proceedings of the 2014 25th IEEE Annual International Symposium on Personal, Indoor, and Mobile Radio Communication, IEEE PIMRC 2014, pp. 502-506, Washington DC, Wash, USA, September 2014.

[16] 3GPP TS 36.212 V10.0.0, "Evolved Universal Terrestrial Radio Access (E-UTRA); Physical Channels and Modulation,” 2010.

[17] 3GPP TS 36.213 V14.1.0, "Evolved Universal Terrestrial Radio Access (E-UTRA); Physical Layer Procedures," 2016.

[18] T. A. Thomas and F. W. Vook, "Improving the performance of the UCA when using LTE codebook feedback," in Proceedings of the 2012 IEEE Wireless Communications and Networking Conference, WCNC 2012, pp. 847-851, Shanghai, China, April 2012.

[19] L. Wu, H. Yang, and D. Wang, "Hadamard transform based codebook design for uniform circular arrays in mobile radio communications," in Proceedings of the 76th IEEE Vehicular Technology Conference, VTC Fall 2012, 5, 1 pages, Quebec City, Canada, September 2012.

[20] A. Cantoni, B. Li, and H. H. Dam, "MU-MIMO performance with a circular base station array and semi-orthogonal user selection," in Proceedings of the TENCON 2015, pp. 1-6, Macao, China, November 2015.

[21] E. Yaacoub, M. Husseini, and H. Ghaziri, "An overview of research topics and challenges for 5G massive MIMO antennas," in Proceedings of the 2016 IEEE Middle East Conference on Antennas and Propagation (MECAP), pp. 1-4, Beirut, Lebanon, September 2016.

[22] V. C. Misra, L. N. Merugu, R. H. Prasad, T. K. Murty, and O. K. Singh, "Beam switching cylindrical array antenna system for communication," Defence Science Journal, vol. 48, no. 4, pp. 403-412, 1998.

[23] T. E. Bogale and L. B. Le, "Massive MIMO and mmWave for 5G wireless HetNet: potential benefits and challenges," IEEE Vehicular Technology Magazine, vol. 11, no. 1, pp. 64-75, 2016.

[24] S. Hur, T. Kim, D. J. Love, J. V. Krogmeier, T. A. Thomas, and A. Ghosh, "Millimeter wave beamforming for wireless backhaul and access in small cell networks," IEEE Transactions on Communications, vol. 61, no. 10, pp. 4391-4403, 2013.

[25] H. Shuangfeng, I. Chih-Lin, X. Zhikun, and C. Rowell, "Largescale antenna systems with hybrid analog and digital beamforming for millimeter wave 5G," IEEE Communications Magazine, vol. 53, no. 1, pp. 186-194, 2015.

[26] S. M. Razavizadeh, M. Ahn, and I. Lee, "Three-dimensional beamforming: a new enabling technology for 5G wireless networks," IEEE Signal Processing Magazine, vol. 31, no. 6, pp. 94-101, 2014.

[27] R. Ahn, M. Weiner, and B. Tomasic, "Digital beamforming in a large conformal phased array antenna for satellite TT\&C - Demonstration," in Proceedings of the IEEE International Symposium on Phased Array Systems and Technology, pp. 807814, Waltham, Mass, USA, 2013.

[28] S. Liu, B. Tomasic, and J. Turtle, "The geodesic dome phased array antenna for satellite operations support - antenna resource management," in Proceedings of the 2007 IEEE Antennas and Propagation Society International Symposium, pp. 3161-3164, Honolulu, Hawaii, USA, June 2007.

[29] B. Tomasic, J. Turtle, S. Liu, R. Schmier, S. Bharj, and P. Oleski, "The geodesic dome phased array antenna for satellite control and communication - Subarray design, development and demonstration," in Proceedings of the 6th IEEE Phased Array Systems and Technology Symposium, Array 2003, pp. 411-416, USA, October 2003. 
[30] McKinsey \& Company, "The road to 5G: The inevitable growth of infrastructure cost," Tech, Media and Telecom Practice, Feb. 2018.

[31] Huawei, 5G Spectrum: Public Policy Position, Industry White Paper, 2017.

[32] A. Basta, A. Blenk, K. Hoffmann, H. J. Morper, M. Hoffmann, and W. Kellerer, "Towards a cost optimal design for a 5G mobile core network based on SDN and NFV,' IEEE Transactions on Network and Service Management, vol. 14, no. 4, pp. 1061-1075, 2017.

[33] C. B. Dietrich, Diversity Antenna Configurations for Handheld Wireless Communication Terminals [Ph.D. thesis], Virginia Polytechnic Institute and State University, Blacksburg, Va, USA, 2000.

[34] 3GPP TR 36.873 V12.2.0, "Study on 3D Channel Model for LTE," June 2015.

[35] R. Aleksiejunas, A. Cesiul, and K. Svirskas, "Spatially consistent LOS/NLOS model for time-varying MIMO channels," in Proceedings of the 2018 Baltic URSI Symposium, URSI 2018, pp. 6164, Poznan, Poland, May 2018.

[36] M. Xiang, Y. Chang, and T. Zeng, "Channel estimation for 3D MIMO system based on LOS/NLOS identification," IET Communications, vol. 13, no. 7, pp. 898-904, 2019.

[37] M. R. Akdeniz, Y. Liu, M. K. Samimi et al., "Millimeter wave channel modeling and cellular capacity evaluation," IEEE Journal on Selected Areas in Communications, vol. 32, no. 6, pp. 1164-1179, 2014.

[38] Y. Cho and J. Kim, "Line-of-sight MIMO channel in millimeterwave beamforming system: modeling and prototype results," in Proceedings of the IEEE 81st Vehicular Technology Conference (VTC-Spring), pp. 1-5, Glasgow, UK, May 2015.

[39] 3GPP TR 38.901 V14.1.1, "Study on channel model for frequencies from 0.5 to $100 \mathrm{GHz}$," July 2017.

[40] S. S. Ioushua and Y. C. Eldar, "A family of hybrid analogdigital beamforming methods for massive MIMO systems," IEEE Transactions on Signal Processing, 2019. 


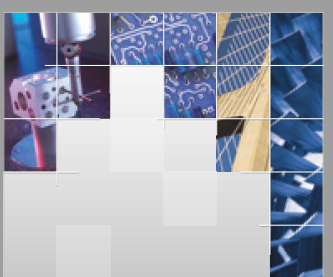

\section{Enfincering}
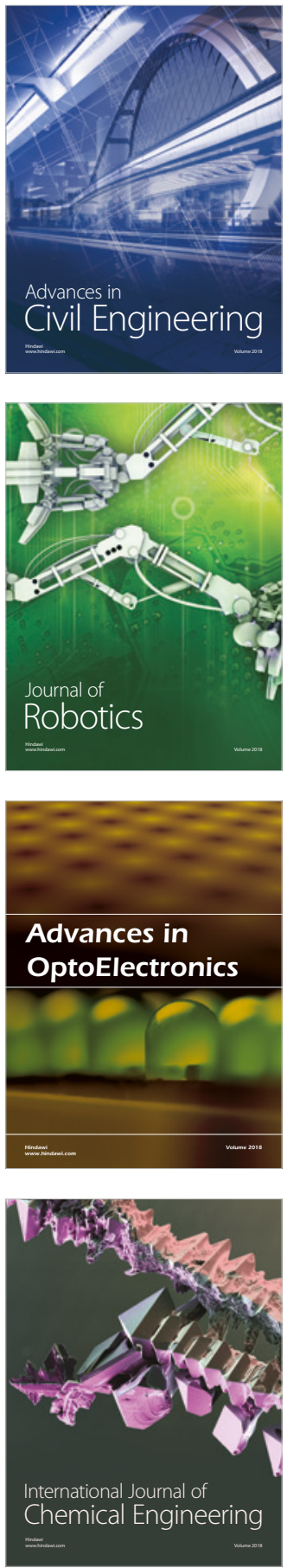

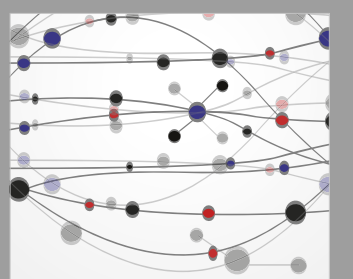

\section{Rotating \\ Machinery}

The Scientific World Journal

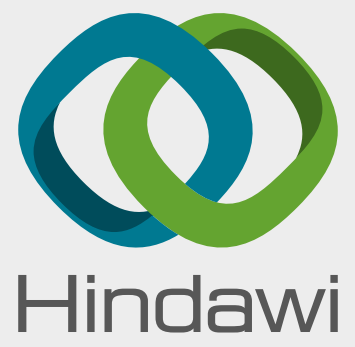

Submit your manuscripts at

www.hindawi.com
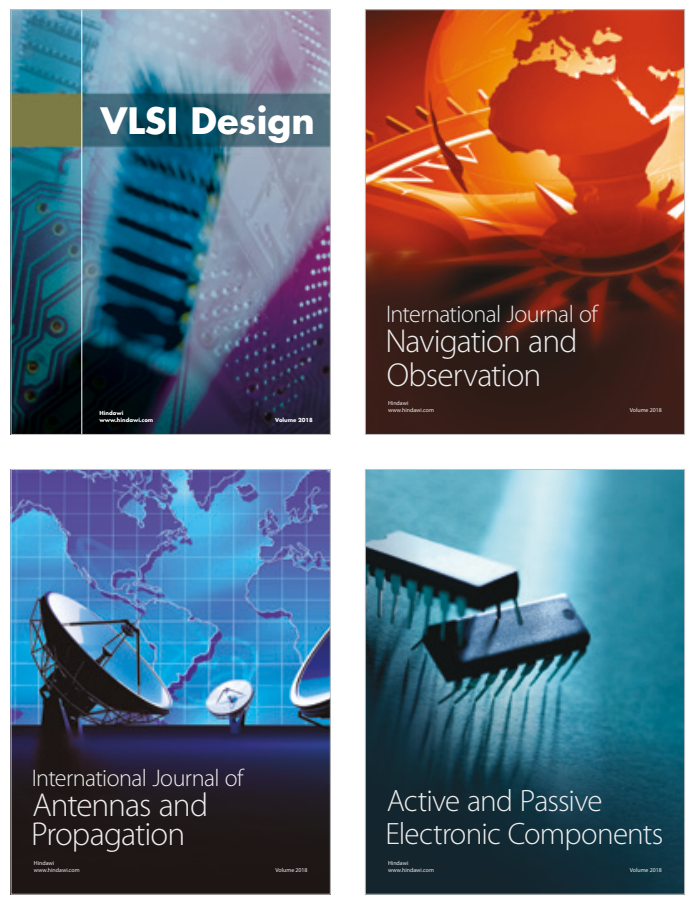
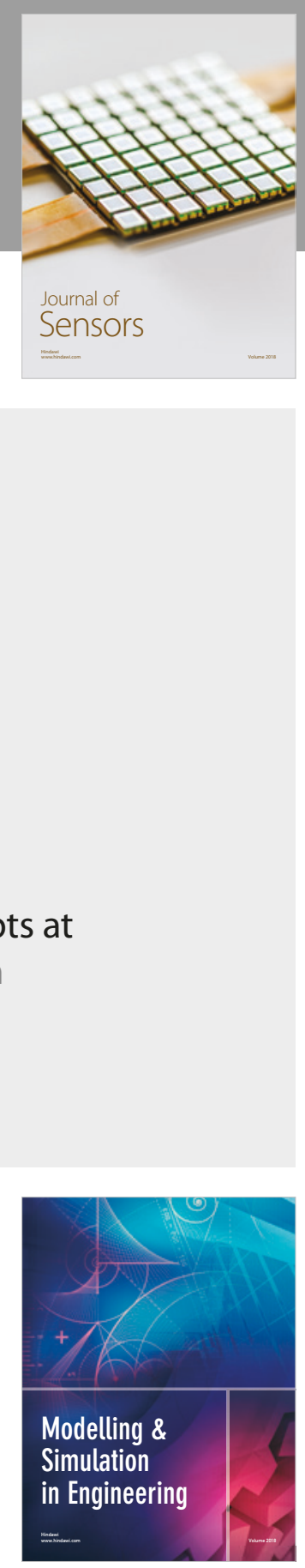

\section{Advances \\ Multimedia}
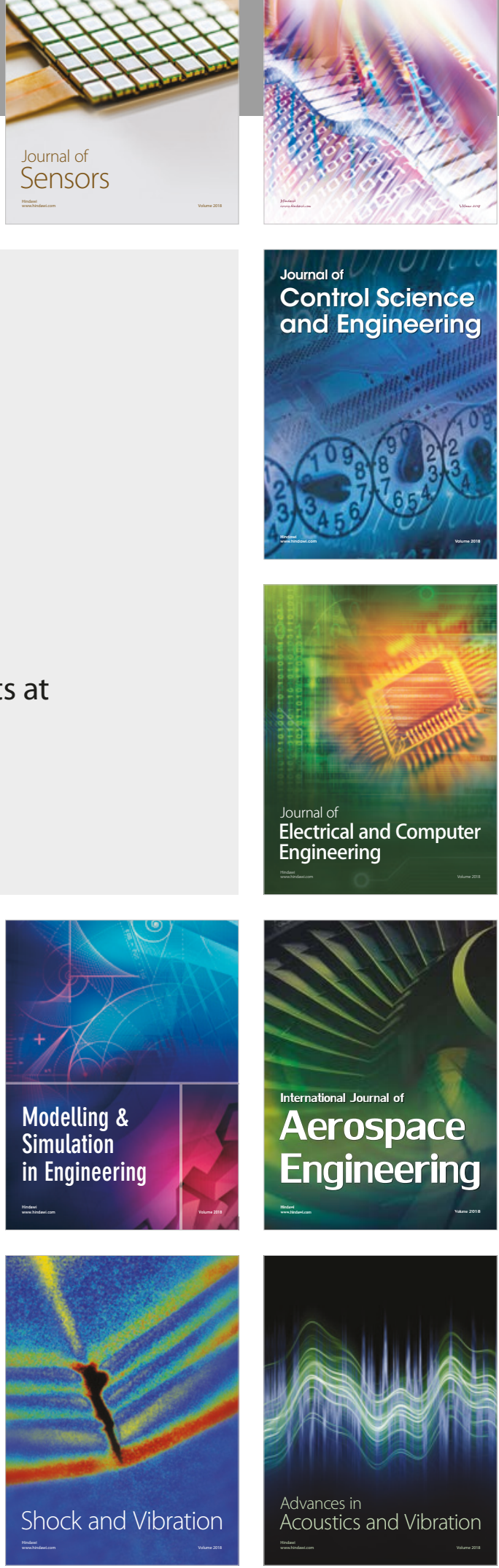